\title{
GUIDELINES FOR IMPROVED EFFECTIVENESS OF AIRBORNE TRAINING OF MILITARY AVIATOR CADETS
}

\author{
Ivaylo NESHEV \\ "Vasil Levski” National Military University, Veliko Tarnovo, Bulgaria \\ neshev_in@abv.bg
}

\begin{abstract}
The paper treats issues relating to the specialized training of officer cadets, particularly future military pilots' airborne training. Having taken into account the new tendencies, the guidelines for conducting airborne training are set forth with view of ensuring their safety during parachute jumps, and subsequently, during flights.
\end{abstract}

KEYWORDS: airborne training, military pilots, effectiveness

\section{Introduction}

The main objectives of the military educational process are identified and defined in the regulatory framework of "Vasil Levski" NMU. They are as follows preparation and conducting of a highly effective educational process based on modern approaches and methods of training; integration in the curricula and lecture fund of adopted standards for achieving a high level of interoperability; study of combat experience from the participation of units of the Bulgarian Army in missions abroad and timely integration of lessons learned in the educational process (Krastev, 2017); improvement of existing educational facilities for conducting quality education in the new range of tasks in operations other than war and post-conflict interoperability.

Training of military pilots must necessarily include parachute jump training (MoD, 1978), so that the skills and habits necessary for bailing out of the aircraft by means of a parachute jump are formed.

The continuous improvement in the different types of aircraft is connected to the continuous increase of flight altitude, distance and speed (MoD, 1998). The improvement of these characteristics is also linked to the enhanced structural complexity of the aircraft. As a result, even though there is an improvement in the technical capabilities of the aircraft, the malfunction index is still positive.

The issues of emergency rescue of airborne personnel influence flight safety (MoD, 1980). In connection to this, the only reliable method of ensuring the survival of the said personnel is bailing out of the aircraft. The adequate assessment of the situation, the timely decisions about bailing out, the psychological and physical preparedness for executing a jump (in that case, catapulting), are of utmost importance for saving the pilot's life, when there are no other rescue methods available.

Thus, the following hypothesis can be posited: the parachute jump training of future pilots can only be effective if there is a methodologically organised interaction between the cadets, and their instructors and lecturers, provided that all of them are equally interested in maintaining a high level of preparedness. 
2. Main Characteristics of Parachute Jump Training

Parachute jump training is a complex of training, educational, technical and technological activities that exert comprehensive influence on cadets in terms of forming permanent knowledge, skills and habits, as well as physical and psychological preparedness for executing parachute jumps.

Naturally, a contradiction exists between the expanding body of knowledge the future pilots need to acquire and the limited resource of practice hours necessary for conducting the parachute jump training.

The improved capabilities of the newgeneration aircraft undisputedly call for high-quality training. This is connected to the intense information flow which is essential in the training, as well as the introduction of new curriculum subject. Last but not least, the obligatory training of the future officers in two majors - a military and a civilian one - is also an important factor behind this need.

The academic subject Basics of Parachute Training is part of the Military Pilot specialization curriculum of the Organisation and Management of Air Force Units on Tactical Level bachelor degree programme (NMU, 2016).

The said academic subject is part of the mandatory corpus of subjects included in the Specialised Military Training section and is taught in the first year.

\section{Structure of Parachute Jump}

\section{Training}

The curriculum includes a single module which covers basic aspects of the subject matter.

The drills are aimed at learning the parachute jumps equipment, the rules for employing the parachute devices, the correct way of folding the parachutes, the rules applicable for jumping in various situations and under various conditions.
The main purpose is that the trainees acquire theoretical awareness and knowledge about equipment and parachute jump techniques, along with practical skills in deploying them ("Vasil Levski" NMU, 2016). Upon completion of the theoretical course in the required volume and the preset sequence of topics, in accordance with the curriculum and this syllabus, the trainees must to know:

- the requirements of the Airborne Jump Field Manual (FM 3-21.220, 2003);

- the purpose and structure of the personal equipment;

- the theory of parachute jumps and actions performed by the jumper in the air and on the ground;

- the protocol for landing on obstacles, under special circumstances and in the event of deployment of the reserve parachute.

Upon completion of the practical training as laid out in the curriculum, the trainees must be able to:

- Perform adequate actions in the air and upon landing;

- Perform daytime medium-altitude induction and training jumps;

- Perform jumps from various types of airplanes and helicopters.

The Basics of Parachute Jumps syllabus includes the execution of two jumps in the first year, an induction and a practice one, respectively (MoD, 1998).

\section{Methodology Aspects}

\subsection{Improving the Theoretical Part}

\section{of the Training}

Taking into account the above factors, instructors should use the following methods while training airborne personnel (Krastev, Simeonov \& Baychev, 1980):

Oral presentation. Oral presentations can be in the form of a narration, when the topic does not require detailed explanation; in the form of an explanation when comparing, and drawing conclusions, etc., 
and, lastly, in the form of a lecture. Lectures aimed at clarifying the specifics of parachute systems deployment should meet special requirements. Unlike other lectures, they contain more description and theoretic elements and, respectively, less narration.

Discussion. Discussion is the training method allowing trainees to answer the supervisor's questions on the given topic and vice versa. During discussions, instructors can put a greater emphasis on the individual approach to any of the cadets, by having them participate in the seminars more actively. This method is constantly used in airborne training. Its shortcoming is the limited opportunity for systematic expounding of content matter and the considerable consumption of study time.

\subsection{Improving the Practical Part of the Training}

Demonstration - The training process should, under all circumstances, be provided with visual aids and should incorporate demonstrations and elucidations. Thus, all exercises in the parachute jump training process should feature all equipment relevant to the topic. For example, when cadets study the construction and mechanics of the parachute, its purpose and characteristics should be explained first, and only after that hands-on familiarization with it and demonstration of its main parts and their interaction should follow (Krastev, Simeonov \& Baychev, 1980).

Practical work - The practice in parachute jump training (examining and learning the equipment, folding and the principles of deployment) is a method that connects theory with practice. For the normal course of practical work, sufficient equipment should be provided, e.g., training parachutes, tools (depending on the method of deployment), folding equipment. The methods for folding a parachute that has missing parts or they are in disrepair should not be taught. Practice folding should not differ from folding a parachute before an actual jump.
An important element of airborne training is the on-the-ground training. It mirrors the sequence of the actual pre-jump preparation. All of the parachutist's actions are learnt and practiced element by element. The whole lesson on the jump training training range should be organised so that the sequence of actions is never compromised.

The abovementioned and the changed conditions under which military units carry out missions call for at least a partial overhaul of the parachute-jump training methodology. This change is also necessitated for by the following factors:

A) The launch into operational use of the new C27J "Spartan" military transport airplane, from which the airborne personnel exit through the side doors and the cargo ramp (MoD, 2008).

B) The continuos participation in multinational exercises and the execution of airborne jumps from NATO partner countries military cargo planes.

Bearing in mind $\mathrm{A}$ and $\mathrm{B}$ above, in elaborating a jump training methodology, special attention should be paid to the following issues:

a. Parachutist's actions in the aircraft;

b. Commands issued by the jumpmaster;

c. Exiting the aircraft;

d. Actions when deploying reserve parachute.

Parachutist's actions in the aircraft:

While in flight, the parachutist should be careful in his actions, should not stand up from his seat and should always watch his static line and obey the commands of the crew and the jumpmaster.

With view of creating habits for correct execution of jumps, in line with the first criterion - Probability of ensuring the safety of the parachutist-parachute system, when practicing jumps from a multiseater military cargo aircraft like the $\mathrm{C} 27$ and its equivalents, completing a training course on mock aircraft is mandatory. Particular attention should be paid to the first lesson: the trainees must be instructed on the 
tactical and technical characteristic of the airplane, focusing on the speed of exiting, the location and type of descent equipment and the seating arrangement of personnel in the airplane. During boarding, the parachutist must be particularly careful as not to open prematurely his parachute and break parts of the equipment. Clothing and webbing must fit properly. Boarding and seating must take place in strict order, where the parachutists must follow the orders of the jumpmaster and the loadmaster. A new development when jumping from $\mathrm{C} 27 \mathrm{~J}$ is that after taking up the designated seats, parachutists must fasten the safety belts. The number of parachutists, boarding and seating in the cargo hatch is determined by the jumpmaster and the crew commander, and is executed by the loadmaster and the jumpmaster (Ministry of Defense, 2008).

The maximum number of parachutists boarding the airplane is 44 (seated in three rows) and 28 (seated in two rows); they are assigned into groups and seated by the loadmaster so as to ensure the balance of the airplane.

When boarding and exiting the airplane, all airborne personnel must follow the commands (verbal and visual signals and the jumpmaster's commands) issued in the cargo hatch. The most important commands are the signals given by the loadmaster and the commands by the jumpmaster (FM 57-1/AFM 2-51, 1981).

The signals given by the loadmaster and the commands given by the jumpmaster are as follows:

Signal "5 (five) minutes" - issued by the loadmaster. At this signal, the jumpmaster sends a command to the inboard stick (the airborne jump personnel in the airplane) to stand up and line up in the order of the jumping (exit from the doors, the ramp), after which the jumpmaster gives the following commands:

Signal "CHECK THE STATIC LINE" - upon receiving this command, parachutists check visually their own static line and grip it with their hands. In the event of jumping from the jump doors, parachutists from the left-hand side hold the static line with the left hand and parachutists from the right-hand side - with their right hand. When jumping from the cargo ramp, the parachutists on the left-hand side grab the rope with their right hand and the parachutists on the right-hand side - with their left hand. After this, each parachutist checks the static line of the man in front of him.

CHECK THE EQUIPMENT Signal upon receiving this command, each parachutist checks: the helmet, the secure fastening of parachute snaps, the secure attachment of reserve parachute and the auxiliary equipment.

SOUND OFF FOR EQUIPMENT CHECK signal - at this command, the parachutists, in a reverse order to their jump order, signal their readiness for jump by tapping the thigh of the parachutist in front of them, duplicating it with the verbal signal "READY". The parachutist to jump first reports to the jumpmaster the readiness of the inboard stick using the ALL READY signal;

1 (ONE) MINUTE signal of the loadmaster: on this signal, the jumpmaster gives a "one minute" command to the inboard stick. Parachutists repeat the command and duplicate it with a verbally. The jumpmaster continues to follow the tack, the exit point and the obstacles along the way of each parachutist to the exit point (jump door, cargo ramp);

30 (THIRTY) SECONDS signal of the loadmaster - on this signal, the jumpmaster gives a 'thirty second' command to the inboard stick. Parachutists repeat the command and duplicate it verbally;

STAND BY signal - at this command the parachutists take position at the exit point;

GO signal of the loadmaster: with this signal the jumpmaster issues a GO command to each parachutist duplicating it with a tap on the shoulder;

NO DROP (ABORT) signal; 
EMERGENCY BAILOUT signal of the loadmaster duplicated verbally (bail out) at this signal, the jumpmaster issues a command to the parachutists to bail out as an inboard stick in the designated order for jumping; the jumpmaster is the last one to bail out. The bailout interval is 1 (one) second. In the event of unattached static line, parachutists (third row of seats aboard the plane), the parachutists attach the static line ropes to the aircraft descent rope and bail out of the plane in the designated jump order.

The increased number of commands calls for the modification and expansion of the training methodology. Consequently, ground preparation in the Parachutist Actions Onboard the Aircraft section should be divided into two parts.

The first part should be Traditional Aircraft Commands Drills. The description of the parachutist's actions onboard and the commands he executes, as described in the Parachute Jump Training Instruction Manual (FM 3-21.220, 2003), can be used as a theoretical basis.

The second part should be Modern Aircraft and NATO Aircraft Commands Drills. In this section, the trainees will practice the procedures in compliance with the relevant modern aircraft instructions.

Exiting the aircraft:

Exiting the aircraft takes place either through the aircraft jump doors or through the rear cargo ramp.

A) Through the jump door - the parachutist puts his left foot in the middle of the lower part of the door, the right foot is backward, the palms are placed on the reserve parachute. The paratrooper's body is at an angle of 45 degrees to the tail of the plane. The parachutist pushes vigorously back with his leading leg while leaping forward, in a tight body position and limbs held next to body;

B) From the rear cargo ramp - at the GO command, the parachutist shuffles towards the end of the ramp while simultaneously dropping the static line and placing his hands on the reserve parachute. The parachutist pushes vigorously back with his leading leg while leaping forward, in a tight body position and limbs held next to body.

The execution of parachute jumps from the cargo ramp is a new addition to the airborne units training. In the past, the exiting process was done mainly by parachute systems with delayed deployment, while nowadays they are mostly performed through the static-line deployment. Another novelty (MoD, 2009) is the placing of the rucksack on the parachutist body - it is now situated below the reserve parachute, on the parachutist's front. This calls for changing the way of exiting the aircraft and a general update of the existing methodology for rear cargo ramp exiting.

\section{Conclusions}

It is logical to assume that future pilots should be prepared for the flight conditions they will face in their career. In view of this, the parachute jump training programme should provide for more sophisticated parachute jumps such as ones executed under adverse weather conditions, during nighttime, over an unfamiliar area, and at ground wind exceeding $6 \mathrm{~m} / \mathrm{s}$.

An analysis of the practice in recent years shows that the scheduled two parachute jumps over the duration of the training cannot satisfy the objective flight crew safety requirements. 


\section{REFERENCES}

FM 3-21.220 (2003). Static Line Parachuting Techniques and Training. Washington. DC: Author.

FM 57-1/AFM 2-51 (1981). US Army/US Air Force Doctrine for Airborne Operations. Washington. DC: Author.

Ivanov, N. (1976). Parachute Jump Training. Sofia: Military Technical Publishing.

Krastev, K. (2017). Modern Approaches in the Training of Military Police Cadets at Vasil Levski National Military University. Proceedings of The $23^{\text {rd }}$ International Conference - The Knowledge-Based Organization. Sibiu, 172-178.

Krastev, S., Simeonov, S. \& Baychev, D. (1980). Parachute Jumps Training Manual. Sofia: Military Technical Publishing.

Ministry of Defense. (1978). Theory and Practice of Parachute Jumps Training. Sofia: Military Technical Publishing.

Ministry of Defense. (1998). Instruction on Parachute Jumps Training, Sofia: Military Technical Publishing. Author.

Ministry of Defense. (2008). Instruction for Parachute Jumps from C-27 J. Sofia:

Ministry of Defense. (2009). Technical Manual - Field Maintenance Manual Including Repair Parts and Special Tools Lists for MC-6 Personnel Parachute System. Sofia: Author.

Vasil Levski National Military University. (2016). Basics of Parachute Jumps Training Syllabus. Veliko Tarnovo: Author. 